\title{
Social Anxiety in a Multiple Sclerosis Clinic Population
}

Külli Poder, Kayhan Ghatavi, John D Fisk, Trudy Campbell, Stephen Kisely, Ian Sarty, Karen Stadnyk, Virender Bhan.

Külli Poder resident Department of Psychiatry, Dalhousie University, Halifax, Nova Scotia, Canada B3H 2E2 Kayhan Ghatavi psychiatrist Peterborough Regional Health Centre, Peterborough, Ontario, Canada K9J 7C6 John D Fisk associate professor Department of Psychiatry, Dalhousie University, Halifax, Nova Scotia, Canada B3H 2E2 Trudy Campbell nurse practitioner Department of Medicine, Dalhousie University, Halifax, Nova Scotia, Canada B3H 2Y9 Stephen Kisely professor of Epidemiology and Community Health, School of Medicine, Griffith University, Meadowbrook Qld 4131, Australia Ian Sarty research assistant Capital Health, Halifax, Nova Scotia, Canada B3H 1V7 Karen Stadnyk data analyst Capital Health, Halifax, Nova Scotia, Canada B3H 1V7 Virender Bhan associate professor Department of Medicine, Dalhousie University, Halifax, Nova Scotia, Canada B3H 2 Y9

Correspondence to: John D Fisk john.fisk@cdha.nshealth.ca

Key words: multiple sclerosis, social anxiety, prevalence, Social Phobia Inventory

Word count: 2,906 


\section{ABSTRACT}

Background: Little is known about the prevalence of social anxiety in multiple sclerosis (MS). This study explored the prevalence of social anxiety and its association with demographic and clinical features in a clinic-attending sample of MS patients living in the community.

Methods: Patients attending the Dalhousie MS Research Unit for regularly scheduled visits during a four month interval were asked to complete the Social Phobia Inventory (SPIN) and the Hospital Anxiety and Depression Scale (HADS), as well as the Mark III version of the Health Utilities Index (HUI) for assessment of perceived health-related quality of life. Neurological disability was determined by neurologist ratings on the Expanded Disability Status Scale (EDSS).

Results: 251 patients agreed to complete self-report scales of symptoms of anxiety and depression and 245 (98\%) provided sufficient data for analysis. 30.6\% $(n=75)$ of the sample had clinically significant social anxiety symptoms as defined by a SPIN threshold of 19. Neurological disability did not differ between these groups. Half of those with social anxiety also had general anxiety (HADSA $\geq 11$ ) and a quarter had depression (HADSD $\geq 11$ ). The severity of social anxiety symptoms was not related to the level of neurological disability, although it was associated with reduced healthrelated quality of life.

Conclusions: Social anxiety is common in persons with MS and contributes to overall morbidity. As social anxiety appears unrelated to the severity of neurological disability, screening for social anxiety symptoms is an important component of comprehensive care for persons with MS. 


\section{INTRODUCTION}

Psychiatric co-morbidity, often of a serious and disabling nature, has long been observed as a prevalent clinical feature among people living with multiple sclerosis (MS). As MS is a chronic neurological condition with a relatively early age of onset, these potentially treatable co-morbid disorders are important targets for detection.[1,2]

Depression has been the predominant focus of research into psychiatric co-morbidity in MS, with an approximate 50\% lifetime prevalence reported.[3] Given the highly co-morbid nature of depression and anxiety in the general population, one would also expect high rates of anxiety disorders in MS. Indeed, several recent studies have demonstrated higher rates of anxiety among people with MS than in the general public.[3-7] Co-morbid anxiety with depression in MS patients has been associated with increased suicidal ideation, alcohol abuse, increased somatic complaints and greater social dysfunction.[4,8,9] To date few studies have explored the prevalence of different subtypes of anxiety disorders, such as social anxiety disorder in individuals with MS.

In the Diagnostic and Statistical Manual of Mental Disorders (DSM-IV-TR),[10] social anxiety disorder is defined as excessive and persistent fear of social and performance situations in which there is the potential for embarrassment. These situations provoke intense anxiety symptoms, often experienced as somatic symptoms such as: palpitations, tremors, sweating, gastrointestinal symptoms, muscle tension and, most typically, blushing. Individuals with social anxiety may avoid social situations as a result. Social anxiety disorder can be particularly disabling with reduced likelihood of employment, social isolation, functional disability and dissatisfaction with life and health. The highly co-morbid nature of social anxiety disorder with depression, panic disorder and alcohol abuse is well established.[11,12] In Canada, the national lifetime and 12 month prevalence of social anxiety disorder have been estimated at $8 \%$ and $3 \%$ 
respectively,[11] while in the province of Nova Scotia, where the current study was based, the 12 month prevalence of social anxiety disorder has been estimated at 4.2\%.[13]

Strict adherence to the DSM-IV-TR does not allow for a diagnosis of social anxiety when the symptoms are considered limited to the general medical condition's social impact (i.e. “secondary social anxiety"). Nevertheless, individuals with chronic physical or mental illnesses who also suffer from excessive levels of social anxiety symptoms have greater clinical and social morbidity.[14-17] In chronic neurological disorders including: spasmodic torticollis (41.3 \%),[14] Parkinson disease (29\%),[17] and essential tremor (22\%),[18] a high prevalence of social anxiety was found when modified DSM criteria for social anxiety disorder were used to allow inclusion of those in whom excessive social anxiety was considered secondary to their medical condition.

This study was designed to determine the prevalence of clinically significant symptoms of social anxiety in a representative clinic sample of people with MS, and to examine its relations to common psychiatric comorbidities, severity of MS symptoms, and health-related quality of life. 


\section{METHODS}

\section{Subjects}

This study was conducted at the Dalhousie Multiple Sclerosis Research Unit (DMSRU) in Halifax, Nova Scotia Canada. The DMSRU is the only specialist clinic serving people with MS in the province of Nova Scotia which has a population approximately 940,000. A patient registry has been maintained at the DMSRU for the past 26 years and linkage with provincial health care records has determined that this registry includes over $80 \%$ of the persons with MS in Nova Scotia.

The study recruited patients with a definite diagnosis of MS,[19] over age 18 who visited the clinic between May 1, 2006 and August 31, 2006, and who provided written informed consent to participate in the study. Patients whose cognitive capacity was considered likely to prevent them from understanding and filling out the questionnaires (i.e. EDSS Mental Functional System score $\geq 2$ and/or clinician judgment) and patients enrolled in clinical trials were excluded. Approval for this study was obtained from the Capital Health Research Ethics Board.

\section{Instruments}

The DMSRU routinely collects sociodemographic and clinical information on each visit and maintains a database of this information. In addition to patient age, gender, education, marital and employment status, MS clinical subtype (relapsing remitting, secondary progressive or primary progressive) and MS status (stable or relapsing), the following routinely collected clinic data were employed. Neurologist ratings on the Expanded Disability Status Scale (EDSS) were used to assess MS related neurological disability.[20] Patient-reported health-related quality of life (HRQoL) was assessed using the Mark 3 version of the Health Utility Index (HUI).[21] This generic measure of health utility provides a single value that represents an 
individual's health state on an interval scale from 0 (dead) to 1 (perfect health). The HUI reflects person preferences and is derived from ratings on eight health attributes including: hearing, speech, ambulation, vision, dexterity, cognition, emotion, and pain. We previously demonstrated that the HUI is a stable and valid measure of HRQoL in MS.[22]

Our study employed the Social Phobia Inventory (SPIN) to assess symptoms of social anxiety.[23] We chose the SPIN as it is a brief screening instrument that can be easily administered in a general medical practice. This 17- item self report questionnaire is available in the public domain. Its items evaluate the fear, avoidance and physiological distress components of social anxiety with severity of each symptom rated on a scale from 0 to 4 such that the total score ranges from 0 to 68. The SPIN has demonstrated good reliability in screening for social anxiety and for measuring treatment outcome in psychiatric outpatients; a score of $\geq 19$ has been found to distinguish between those with and without social anxiety disorder.[23,24]

We used the Hospital Anxiety and Depression Scale (HADS), a 14-item self administered questionnaire designed to assess symptoms of general anxiety (HADSA) and depression (HADSD) in medically ill populations.[25] Studies using the HADS with MS patients have assigned cut off scores ranging from 8 to 11.[6,7,9,26,27] We chose to use the more conservative cut off score of $\geq 11$, as suggested by the original authors, to identify the probable presence of depression and/or anxiety disorder.[25]

The CAGE questionnaire was used to screen for potential co-morbid alcohol abuse. This four item screening tool was developed to detect problematic alcohol use and has been validated in ambulatory medical patients.[28] Cut off scores $\geq 2$ suggest the presence of alcohol abuse or dependence.[29] 


\section{Statistical analysis}

Patients with a SPIN score of $\geq 19$ were considered to have clinically significant symptoms of social anxiety. These high (social anxiety) and low (no social anxiety) SPIN groups were compared using chi-square analyses, t-tests and Mann Whitney $\mathrm{U}$ tests for categorical, continuous and ordered data respectively with a p value of $<.05$ chosen to indicate statistical significance. Spearman's correlation coefficients, with pairwise exclusion of missing cases, examined the associations between the SPIN, HADSA, HADSD, EDSS, and HUI. We considered correlations below 0.30 as evidence of divergent validity, correlations from 0.30 to 0.59 as evidence of moderate convergent validity, and correlations exceeding 0.59 as strong evidence of convergent validity.[30]

\section{RESULTS}

277 patients met the inclusion criteria and were asked to participate in the study. Of these, 26 declined and 251 (91\%) gave their consent to participate. Data from 6 (2\%) participants were excluded from the analyses, because they failed to complete two or more questions on the SPIN, leaving data from 245 in the study sample. For patients who met the inclusion criteria, there were no statistically significant differences in age, sex, EDSS, or classification of MS between those with $(n=245)$ and without $(n=32)$ complete data available for analyses.

The prevalence of social anxiety in the study sample was $30.6 \%(n=75)$, based on a SPIN cut off score $\geq 19$. Table 1 presents the sociodemographic and clinical characteristics of the low and high SPIN subgroups. 
Table 1: Sociodemographic and clinical characteristics of participants.

\begin{tabular}{|c|c|c|c|}
\hline & Total & $\mathrm{SPIN}<19$ & $\mathrm{SPIN} \geq 19$ \\
\hline Variable & $(n=245)$ & $(n=170)$ & $(n=75)$ \\
\hline Age & $46.1 \pm 10.6$ & $46.3 \pm 10.9$ & $45.7 \pm 10.1$ \\
\hline Gender (Female) & $200(82 \%)$ & $134(79 \%)$ & $66(88 \%)$ \\
\hline Married or common law & $181(74 \%)$ & 127 (75\%) & $54(72 \%)$ \\
\hline Education (years)* & $14.0 \pm 2.7$ & $14.3 \pm 2.7$ & $13.2 \pm 2.6$ \\
\hline \multicolumn{4}{|l|}{ Employment status: † } \\
\hline Employed & $106(43 \%)$ & $83(49 \%)$ & $23(31 \%)$ \\
\hline Disability pension & 83(34\%) & $52(31 \%)$ & $31(41 \%)$ \\
\hline Other & $56(23 \%)$ & $35(21 \%)$ & $21(28 \%)$ \\
\hline Mean \pm SD years of MS (median) & $14.4 \pm 9.2(14.0)$ & $14.6 \pm 9.7(14.0)$ & $14.0 \pm 7.8(13.0)$ \\
\hline \multicolumn{4}{|l|}{ Class of MS: } \\
\hline Relapsing Remitting & $172(70 \%)$ & $119(70 \%)$ & $53(71 \%)$ \\
\hline Secondary Progressive & $57(23 \%)$ & $41(24 \%)$ & $16(21 \%)$ \\
\hline Primary Progressive & $16(7 \%)$ & $10(6 \%)$ & $6(8 \%)$ \\
\hline EDSS mean \pm SD (median) & $3.2 \pm 2.2(2.5)$ & $3.1 \pm 2.1(2.5)$ & $3.5 \pm 2.2(3.0)$ \\
\hline \multicolumn{4}{|l|}{ MS status: $\ddagger$} \\
\hline Stable or improving & $193(79 \%)$ & $141(83 \%)$ & 52 (69\%) \\
\hline Acute attack or progressive & $51(21 \%)$ & $28(17 \%)$ & $23(31 \%)$ \\
\hline
\end{tabular}

${ }^{*} p=0.007, \dagger p=0.03, \ddagger p=0.012$ 
Age, gender, MS duration, MS subtype, and neurological disability, as measured by the EDSS, did not differ between those with and without social anxiety. Those with social anxiety had on average one year less of education, although both groups were relatively well educated. A smaller proportion of those with social anxiety had stable MS at the time of the study. Patients with social anxiety were also less likely to be employed and more likely to be receiving disability pensions at the time of the study.

Table 2 presents self-reported symptoms of depression and general anxiety as well as selfreported health-related quality of life.

Table 2: Self reported psychosocial aspects of patients with high and low SPIN scores

$\begin{array}{lccc}\text { Instrument } & \text { Total } & \text { SPIN }<\mathbf{1 9} & \text { SPIN } \geq \mathbf{1 9} \\ \text { HUI mean } \pm \mathrm{SD}^{*}(\mathrm{n}=237) & 0.57 \pm 0.32 & 0.67 \pm 0.27 & 0.35 \pm 0.30 \\ \text { HADS anxiety mean } \pm \mathrm{SD}^{*}(\mathrm{n}=233) & 7.4 \pm 4.2 & 5.9 \pm 3.2 & 10.7 \pm 4.1 \\ \text { HADS depression mean } \pm \mathrm{SD}^{*}(\mathrm{n}=236) & 4.8 \pm 3.9 & 3.4 \pm 2.9 & 8.1 \pm 4.0 \\ \text { Anxiety (HADSA } \geq 11)^{*} & 49 / 233(21 \%) & 13 / 161(8 \%) & 36 / 72(50 \%) \\ \text { Depression (HADSD } \geq 11) * & 22 / 236(9 \%) & 3 / 166(2 \%) & 19 / 70(27 \%) \\ \text { Alcohol abuse }(\mathrm{CAGE} \geq 2) & 9 / 233(4 \%) & 5 / 164(3 \%) & 4 / 69(6 \%) \\ & & & \end{array}$

$* \mathrm{p}<0.001$

Mean scores for symptoms of both depression (HADSD) and general anxiety (HADSA) were higher in those with social anxiety, while health-related quality of life, as measured by the HUI, was lower. Using HADS cut-off scores to identify patients with depression and general anxiety demonstrated high rates of co-morbid social anxiety with both general anxiety (36/49= 73\%) and depression (19/22 = 86\%). However, when the SPIN was used to identify patients 
reporting significant social anxiety symptoms, their rates of co-morbid general anxiety (36/75 = 48\%) and depression (25\%) were relatively less. There was no association between alcohol abuse, as identified by subjects' responses to the CAGE, and the presence of social anxiety. Associations between self-reported social anxiety symptoms, symptoms of co-morbid psychiatric symptoms, health-related quality of life and neurological disability were also examined via correlations (Table 3).

Table 3. Spearman correlation coefficients between SPIN, HADS, EDSS and HUI SPIN HADSA HADSD HUI HADSA $\quad .59 \dagger$ HADSD $\quad .56 \dagger \quad .57 \dagger$ HUI* $\quad-.40 \dagger \quad-.38 \dagger \quad-.65 \dagger$

$\begin{array}{lllll}\text { EDSS } & 0.07 & 0.02 & .36 \dagger & -.58 \dagger\end{array}$

* Negative correlations for HUI reflect that the index is calculated between 1 (perfect health) and 0 (death).

$\dagger$ Correlation is significant at the 0.01 level (2-tailed).

Moderate correlations were found between the SPIN and the HADSA, HADSD and HUI scores; whereas a low and statistically non-significant correlation was found between SPIN scores and ratings of neurological disability on the EDSS. Nevertheless, greater neurological disability (EDSS) had a moderate association with increased symptoms of depression (HADSD) and with reduced quality of life (HUI). 


\section{DISCUSSION}

We found that just over $30 \%$ of a representative sample of individuals presenting for regularly scheduled visits at a regional specialized MS clinic reported clinically significant symptoms of social anxiety. This point prevalence estimate is much higher than the estimated prevalence of social anxiety in the general and regional Canadian population.[11,13] and is in keeping with estimates of social anxiety reported in other chronic neurologic disorders (41.3\%,[14] 29\%,[17] 22\%[18]). However, our prevalence estimate is also higher than other recently published prevalence estimates for MS patients that have been based on the use of the Structured Clinical Interview for DSM IV (SCID) (2.1\%,[4] 2\%[5]). In part, these lower prevalence estimates may reflect the inclusion of only those individuals with relapsing remitting MS and milder neurological disability.[5] This difference in prevalence estimate may also reflect use of the SCID as a case-finding instrument, as per DSM [10], as opposed to our use of the SPIN to identify patients reporting clinically significant symptoms of social anxiety. Lower prevalence of social anxiety might be expected in medically ill subjects when using the SCID as it excludes from consideration those social anxiety symptoms associated with a medical condition (i.e. secondary social anxiety). In contrast, the SPIN is a screening tool that allows for the inclusion of cases with secondary social anxiety as a consequence of the individual's health status.

The lack of an association between severity of self-reported social anxiety symptoms and the severity of either physician-rated neurological disability or the duration from onset of MS symptoms might be considered an unexpected finding. However, social anxiety may well be attributable to the presence of specific neurologic symptoms (e.g. tremor, dysarthria, bowel and bladder dysfunction) that are likely to lead to anxiety in social situations, rather than to overall disability. It is also possible that social anxiety in persons with MS is more associated with the 
anticipated or perceived unpredictability of symptoms rather than with persistent disability and reduced ambulation. Our finding that proportionally fewer patients with social anxiety had stable MS at the time of the study is consistent with this possibility as is the previously reported association of general anxiety with MS exacerbations.[31] Further studies will be needed to explore the possible causal relations between specific MS symptoms and social anxiety.

Social anxiety was associated with poorer self-reported health-related quality of life. This is consistent with general population studies,[11,12] and studies of MS patients which demonstrate a significant association between general anxiety, depression, and patient perceived health-related quality of life.[26] Our study did not find an association between social anxiety and neurological disability, but did confirm our previously reported association between the EDSS and HUI.[22] Thus, our current findings indicate that social anxiety and neurological disability make significant but independent contributions to self-reported health-related quality of life for MS patients.

Our high prevalence of co-morbid general anxiety and depression among those with social anxiety is consistent with general population studies.[12] While our prevalence estimate of social anxiety was higher than that of either general anxiety or depression, it is difficult to make direct comparisons and draw meaningful conclusions about such differential prevalence rates when different measures were used to obtain them. Though studies have suggested that social anxiety may precede other psychiatric co-morbidities in the general population,[11,15] our crosssectional study could not address this possibility.

Importantly, alcohol abuse, as measured with CAGE, was not significantly higher in the social anxiety group, and no more prevalent in our MS sample than in the general Canadian population when measured by the same instrument.[32] Alcohol abuse has been associated with social anxiety in other general population studies,[11,33] and it has been suggested that increased 
alcohol related problems in socially anxious people reflect alcohol use in high risk situations rather than increased consumption.[34] While our lack of an association between alcohol abuse and social anxiety may reflect insensitivity of the CAGE for socially anxious individuals it may also be that alcohol use does not serve to manage the symptoms of social anxiety in persons with MS and that its use may exacerbate the underlying MS symptoms that lead to social anxiety for some.

We must acknowledge a number of limitations to our study. First, our cross-sectional design limited our ability to make causal inferences from associations. We did not address the question of whether social anxiety symptoms developed before or after the onset of MS, and we did not attempt to determine the extent to which social anxiety symptoms were secondary to MS. Secondly, this study was not designed to identify “cases” of social anxiety disorder according to standardized psychiatric nomenclature, although we were able to identify patients who reported clinically significant symptoms of social anxiety. The use of self-report instruments for identification of “cases” contributes to information bias, in that prevalence estimates will vary across studies depending on the instruments used and the cut-points chosen. For this reason, we chose to use the cut-points identified by the test developers as indicative of "caseness" for all self-report instruments. Finally, the generalizability of findings, arising from a convenience sample of consecutive clinic-attending patients, must also be questioned. Nonetheless, the Dalhousie MS Research Unit is the only specialist MS clinic in the region, and it serves approximately $80 \%$ of the individuals with MS in Provincial population; therefore, our study sample can be considered representative of those clinic-attending patients with MS in Nova Scotia. 


\section{CONCLUSIONS}

Clinically significant symptoms of social anxiety are highly prevalent among persons with MS and were present in almost one third of our cross sectional sample of clinic-attending patients. Social anxiety was associated with lower self-reported health-related quality of life as well as with generalized anxiety and depression, but was not associated with MS severity or duration. Social anxiety may contribute to the overall morbidity of MS patients if left unrecognized and untreated. Social anxiety is potentially treatable and such treatment holds promise for improving the quality of life for a significant proportion of people living with MS. Clinicians should consider screening for symptoms of social anxiety, especially as MS severity or duration are not good indicators of its likely presence or severity. Further study of the causes, optimal screening methods, and potential treatments of social anxiety symptoms in MS patients is warranted.

\section{COMPETING INTERESTS}

All authors declare that the answer to the questions on your competing interest form are all No and therefore have nothing to declare.

\section{FUNDING}

This study was funded by a grant from the Dalhousie University Department of Psychiatry Research fund. Additional support was provided by the Nova Scotia Department of Health. All authors contributions are independent of the funding sources. 


\section{COPYRIGHT}

The Corresponding Author has the right to grant on behalf of all authors and does grant on behalf of all authors, an exclusive licence (or non exclusive for government employees) on a worldwide basis to the BMJ Publishing Group Ltd and its Licensees to permit this article (if accepted) to be published in JNNP and any other BMJPGL products to exploit all subsidiary rights, as set out in our licence. 


\section{REFERENCES}

1. Rogers J, Bland R. Psychiatric manifestations of multiple sclerosis: a review. Can $J$ Psychiatry 1996;41:441-445.

2. Feinstein A. The neuropsychiatry of multiple sclerosis. Can J Psychiatry 2004;49:157-163.

3. Siegert RJ, Abernethy DA. Depression in multiple sclerosis: a review. J Neurol Neurosurg Psychiatry 2005;76:469-475.

4. Korostil M, Feinstein A. Anxiety disorders and their clinical correlates in multiple sclerosis patients. Mult Scler 2007;13: 67-72.

5. Galeazzi GM, Ferrari S, Giaroli G, et al. Psychiatric disorders and depression in multiple sclerosis outpatients: impact of disability and interferon beta therapy. Neurol Sci 2005;26:255-262.

6. Janssens AC, van Doorn PA, de Boer JB, et al. Anxiety and depression influence the relation between disability status and quality of life in multiple sclerosis. Mult Scler 2003;9:397-403.

7. Smith SJ, Young CA. The role of affect on the perception of disability in multiple sclerosis. Clin Rehabil 2000;14:50-54.

8. Feinstein A, O’Connor P, Gray T, et al. The effects of anxiety on psychiatric morbidity in patients with multiple sclerosis. Mult Scler 1999;5:323-326.

9. Feinstein A. An examination of suicidal intent in patients with multiple sclerosis. Neurology 2002;59:674-678.

10. America Psychiatric Association. Diagnostic and Statistical manual of Mental Disorders, Fourth Edition, Text Revision. Washington, DC 2000;450-456.

11. Shields M. Social anxiety disorder- beyond shyness. In: Statistics Canada Catalogue 82-003, Supplement to Health Reports 2004;15:47-81. 
12. Cramer V, Torgersen S, Kringlen E. Quality of life and anxiety disorders: a population study. J Nerv Ment Dis 2005;193:196-202.

13. Nova Scotia Department of Health. Social Anxiety in Nova Scotia. Canadian Community Health Survey Cycle 1.2 Report 2; June 2004.

14. Gündel H, Wolfe A, Xidara V, et al. Social phobia in spasmodic torticollis. J Neurol Neurosurg Psychiatry 2001;71:499-504.

15. Moutier CY, Stein MB. The history, epidemiology, and differential diagnosis of social anxiety disorder. J Clin Psychiatry 1999;60(suppl 9):4-8.

16. Oberlander EL, Schneier FR, Liebowitz MR. Physical disability and social phobia. J Clin Psychopharmacol 1994;14:136-43.

17. Stein MB, Heuser IJ, Juncos JL, et al. Anxiety disorders in patients with Parkinson's disease. Am J Psychiatry 1990;147:217-220.

18. Schneier FR, Barnes LF, Albert SM, et al. Characteristics of social phobia among persons with essential tremor. J Clin Psychiatry 2001;62:367-372.

19. Polman CH, Reingold SC, Edan G, et al. Diagnostic criteria for multiple sclerosis: 2005 revisions to the “McDonald Criteria”. Ann Neurol 2005;58:840-846.

20. Kurtzke JF. Rating neurologic impairment in multiple sclerosis: an expanded disability status scale (EDSS). Neurology 1983;33:1444-1452.

21. Feeney DH, Torrance GW, Furlong WJ. Health Utilities Index. In: Spilker B, ed. Quality of life and pharmacoeconomics in clinical trials. $2^{\text {nd }}$ ed. Philadelphia: Lippincott-Raven Pub. 1996:239-252.

22. Fisk JD, Brown MG, Sketris IS, et al. A comparison of health utility measures for the evaluation of multiple sclerosis treatments. J Neurol Neurosurg Psychiatry 2005;76:58-63. 
23. Connor KM, Davidson JR, Churchill LE, et al. Psychometric properties of the Social Phobia Inventory (SPIN). B J Psychiatry 2000;176:379-386.

24. Antony MM, Coons MJ, McCabe RE, et al. Psychometric properties of the social phobia inventory: further evaluation. Behav Res Ther 2006;44:1177-1185.

25. Zigmond AS, Snaith RP. The hospital anxiety and depression scale. Acta Psychiatr Scand 1983;67:361-70.

26. Lester K, Stepleman L, Hughes M. The association of illness severity, self-reported cognitive impairment, and perceived illness management with depression and anxiety in a multiple sclerosis clinic population. J Behav Med 2007;30:177-186.

27. Hakim EA, Bakheit AM, Bryant TN, et al. The social impact of multiple sclerosis--a study of 305 patients and their relatives. Disabil Rehabil. 2000;22:288-293.

28. Dhalla S, Kopec JA. The CAGE questionnaire for alcohol misuse: a review of reliability and validity studies. Clin Invest Med 2007;30:33-41.

29. Ewing JA. Detecting alcoholism. The CAGE questionnaire. JAMA 1984;252:1905-1907.

30. Nunnally JC, Bernstein IH. Psychometric Theory, 3rd ed. New York: McGraw-Hill Inc. 1994:92-94.

31. McCabe MP. Mood and self-esteem of persons with multiple sclerosis following an exacerbation. J Psychosom Res 2005;59:161-166.

32. Poulin C, Webster I, Single E. Alcohol disorders in Canada as indicated by the CAGE questionnaire. CMAJ 1997;157:1543-1545.

33. Katzelnick DJ, Kobak KA, DeLeire T, et al. Impact of generalized social anxiety disorder in managed care. Am J Psychiatry 2001;158:1999-2007. 
34. Buckner JD, Schmidt NB, Eggleston AM. Social anxiety and problematic alcohol consumption: the mediating role of drinking motives and situations. Behav Ther 2006;37:381391. 